\title{
Age and Gender Based Organisation of Shelter Homes using Convolutional Neural Networks
}

\author{
Shravya Suresh, Sneha Venkatesh, Vidya Shree S, Hemalatha V R, T Vijaya Kumar
}

\begin{abstract}
The number of abandoned, homeless and poor people have increased drastically in the recent days. Allotting these people to different shelter home is a very difficult task because volunteers in NGO have to do all the work manually and homeless people don't have valid documentation regarding their Age and Gender. Volunteers usually estimate the person's Age and Gender on the basis of naked eye estimation but this estimation or prediction sometimes will not be accurate. This problematic situation can be solved by using Deep Learning algorithm like Convolutional Neural Network (CNN). So in our project, we use CNN algorithm to estimate the Age and Gender from the facial image which proves to be a challenging task for a machine due to the high extent of variability, lighting and other supporting conditions. The system proposes building a model which has multiple convolutional layers along with dropout and maxpooling layers in between. The proposed model has been trained on UTKFace dataset and Fairface dataset. The proposed system aims to produce a high accuracy in allotting the right shelter home for people under various Age and Gender. The web application also accepts donations from the users visiting the website who are willing to help the shelter home residents.
\end{abstract}

Keywords: Age Prediction, Gender Prediction Convolutional Neural Networks, Deep Learning, Shelter Home Organization.

\section{INTRODUCTION}

In recent days, the number of abandoned, homeless and poor people have increased drastically. It's not a rare sight in a country like India where there are hundreds and probably thousands of such people who live in poor conditions and have nowhere to go. For such people, organisations and even the government have come up with plans of shelter homes that provide basic necessities to these underprivileged people and

Manuscript received on July 20, 2021.

Revised Manuscript received on July 25, 2021.

Manuscript published on August 30, 2021.

* Correspondence Author

Shravya Suresh, Student, Department of Computer Science and Engineering, Bangalore Institute of Technology, Bengaluru, India. Email: shravya995@gmail.com

Sneha Venkatesh, Student, Department of Computer Science and Engineering, Bangalore Institute of Technology, Bengaluru, India. Email: snehavenkatesh10@gmail.com

Vidya Shree S*, Student, Department of Computer Science and Engineering, Bangalore Institute of Technology, Bengaluru, India. Email: vidyashree7499@gmail.com

Hemalatha V R, Student, Department of Computer Science and Engineering, Bangalore Institute of Technology, Bengaluru, India. Email: hemaprasad1899@gmail.com

Dr. T Vijaya Kumar, Associate Professor, , Department of Computer Science and Engineering, Bangalore Institute of Technology, Bengaluru, India. Email: tvijaykumar@bit-bangalore.edu.in

(C) The Authors. Published by Blue Eyes Intelligence Engineering and Sciences Publication (BEIESP). This is an open access article under the CC BY-NC-ND license (http://creativecommons.org/licenses/by-nc-nd/4.0/) give them new and better hope in life. Every human deserves a good quality of life and basic living conditions.

In this paper, we have designed an Age and Gender based organisation of homeless people into different shelter homes using Convolutional Neural Networks (CNN). CNN is mainly used in image recognition, image classifications, object detections, face recognition etc. In order to train and test a deep learning CNN model, each input image is passed through a series of convolution layers with filters, Pooling, fully connected layers and apply activation function to classify an object with probabilistic values between 0 and 1 .

This specific paper involves deep learning model with five convolutional layers, two max-pool layers, two dropout layers and two fully connected layers. The dataset used for this purpose is a combination of UTKFace and Fairface dataset. Using this model, we have predicted the Age and Gender of homeless people by inputting their facial image and these values are used in classifying people based on their Age and Gender and decide which shelter home they should be allotted to.

\section{RELATED WORK}

For decades, researchers have studied age and gender classification. Multiple approaches have been employed over the years to address this issue, with various degrees of complexity.

The network architecture used in [1] is designed to be relatively shallow in order to avoid overfitting the data. They used cutting-edge architecture to actually increase precision by stacking the determination of age with gender. They could have boosted SGD performance by lowering the amount of fully connected layers to one, so that accuracy could have been improved. Similar work can be seen in [2], where they have built a smaller network, to reduce the risk of overfitting as well as to solve the nature of the problems in attempting to solve age classification. But mistakes frequently occurred when adience images of babies were considered for age estimation.

In [3] a new CNN architecture like RoR (Residual of Residual networks) is considered which takes high-resolution facial images i.e., firstly pre-training was done using Imagenet to avoid overfitting and then

fine tuning using IMDB-WIKI-101 dataset. More recently in [4], they have used VGG-16 Architecture on ImageNet to build a DEX technique for age estimation, both real and apparent, and to add the Residual DEX to further improve the DEX Model.

Published By:

Blue Eyes Intelligence Engineering and Sciences Publication

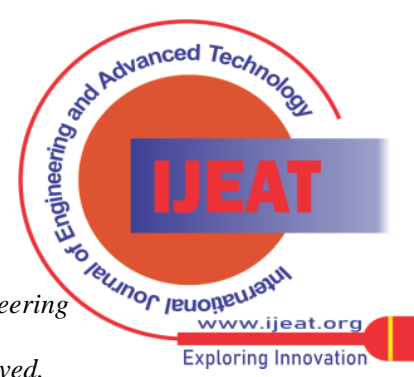


The author of [5] has deployed a deep neural network architecture incorporating CAS-PEAL and FEI for pre-training. This design uses a practical alignment technique based on 3-D facial modeling to improve accuracy and reduce error rates. The Caffe deep learning framework is utilized in [6] to determine the age and gender of the camera images. For GoogLeNet's learning, they used the Adience dataset. To improve training procedures, asynchronous stochastic gradient descent with several GPUs is used.

A rudimentary neural network model [7] is included, which employs best techniques such as SVM, KNN, and Haar cascade to improve age prediction accuracy. They used the TensorFlow framework and the Keras open-source deep learning package.[8] and [9] used VGG19 and VGGFace models that have been pre-trained. To boost accuracy, they altered some training settings. [8] and [9] have attained a high degree of precision.

They used the Google and IMDB datasets in [10] and used the Wrinkle analysis, Adam optimizer, and Haar cascade algorithm, similar to [7], which delivered the maximum level of precision and accuracy.

\section{PROPOSED SYSTEM}

The system proposes an Age and Gender based classification model which helps allocate homeless people to shelter homes. The model has network architecture of 7 layers (5 convoluted and 2 fully connected layers) convolutional layers. The main objectives to be achieved are:

- To design a web application for the Shelter homes.

- To build an Age and Gender based classification model using CNN.

- To minimize the work done by volunteers manually and reduce the paper work involved.

- To implement the concept of automation of work.

\section{SYSTEM ARCHITECTURE}

In this system, the system architecture represents few of the important blocks. It starts with the integration and importing of datasets, UTKFace and Fairface which is followed by the preprocessing of the integrated dataset. The project then goes on to the phase of building the model and training the same. For building the CNN model, we are using various build-in functions present in python. Several layers are built (5 conv +2 FC) and trained on the dataset for about 60 epochs, using the Adam optimizer with a learning rate of 0.25 .

From the user interface, one can either login as admin and request to add a new person into the shelter home by accepting and passing his details into the CNN model for Age and Gender prediction which is further allocate on of the shelter homes to the person based on their respective Age and Gender, or one can signup/login as a user and choose to make donations or suggest a new entry into the shelter home by providing entry level details of the new entry which will be further taken over by the admin for further admission process.
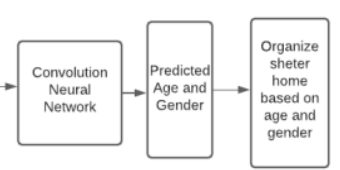

Fig.2 Image Preprocessing

\section{B. CNN Model}

A CNN model is built to predict the Age and Gender of the person using the facial images. Here the model takes images as input and predicts Age and Gender and hence, a CNN architecture has to be built consisting of a number of convoluted layers with a few max pooling and drop out layers in between. Once the CNN model is built, this model should be trained so that, it can predict the useful output for our project. Here, dataset which is preprocessed in the previous module is used to train the model. A learning rate of 0.25 was used and was run 60 epochs to train the model. Once the model is trained it will be tested against different images which will estimate Age and Gender, which is then used to categorize people into different shelter homes.

\section{Login and Registration Module.}

The admin can login only with the valid admin credentials. If the user tries logging In, alert message will be displayed. If the user is already registered, he/she can login directly with the proper credentials or else, he/she can also choose to sign up/Register in case they are new to the system.

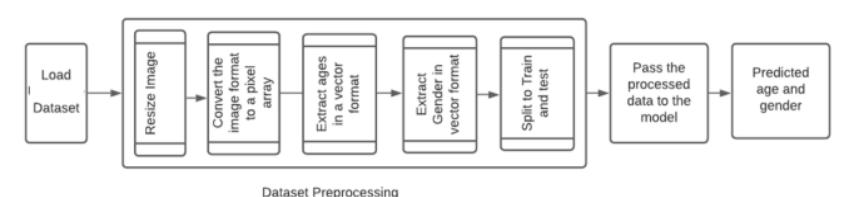

Fig. 3 Dataset Preprocessing

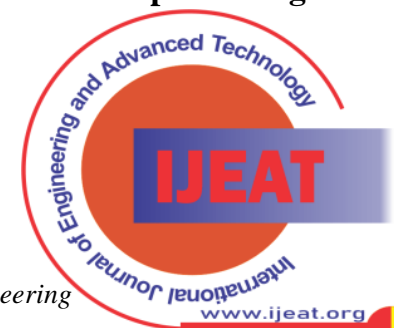
ed. 


\section{User Module}

User has to input the basic details if he/she wishes to admit a new person to shelter home. This information will be directly sent to the admin for further verification and admission.

\section{E. Admin Module}

The details entered by the user to admit a new person has to be verified by the admin. Admin can also input the details for admitting new person to shelter homes.

\section{F. Donation Module}

The details entered by the user to admit a new person has to be verified by the admin. Admin can also input the details for admitting new person to shelter homes.

\section{ALGORITHM}

\section{A. CNN Algorithm}

Input: UTKface and FairFace Dataset, Test image

Output: Predicted age and gender

Step 1: Preprocess the input image (crop the image and resize to $48 * 48 * 3)$.

Step 2: Prepare the model.

Step 3: Run the model for 60 epochs and save the best model with least loss and highest accuracy metrics.

Step 4: Input the test image and Preprocess the test image to fit the model.

Step 5: Pass the above preprocessed image through face detection algorithm and crop out human face from the image.

Step 6: Pass the prepared test image to the model and get the age. If the gender output is greater than 0.5 pass the gender as female else pass it as male.

Step 7:

If (age is less than 10 allocate to Category1\}

Else If (age is greater than 55) \{allocate to Category2\}

Else If (age is greater than 10 and less than 18)

\{

If(gender=Male) $\{$ allocate to Category3 $\}$

else allocate to Category4

\}

else \{

If(gender=Male) $\{$ allocate to Category5

else $\{$ allocate to Category6

\}

In the above algorithm, by Category $1,2,3,4,5$, and 6 we mean the different shelter homes allocate based on Age and Gender.

We have also used the Haar Cascade algorithm popularly known as Viola Jones algorithm for facial recognition. It works by calculating Haar features, creating integral images, uses the Adaboost method to get the best features and later implements the cascading classifier to find positive and negative objects (i.e., Facial features).

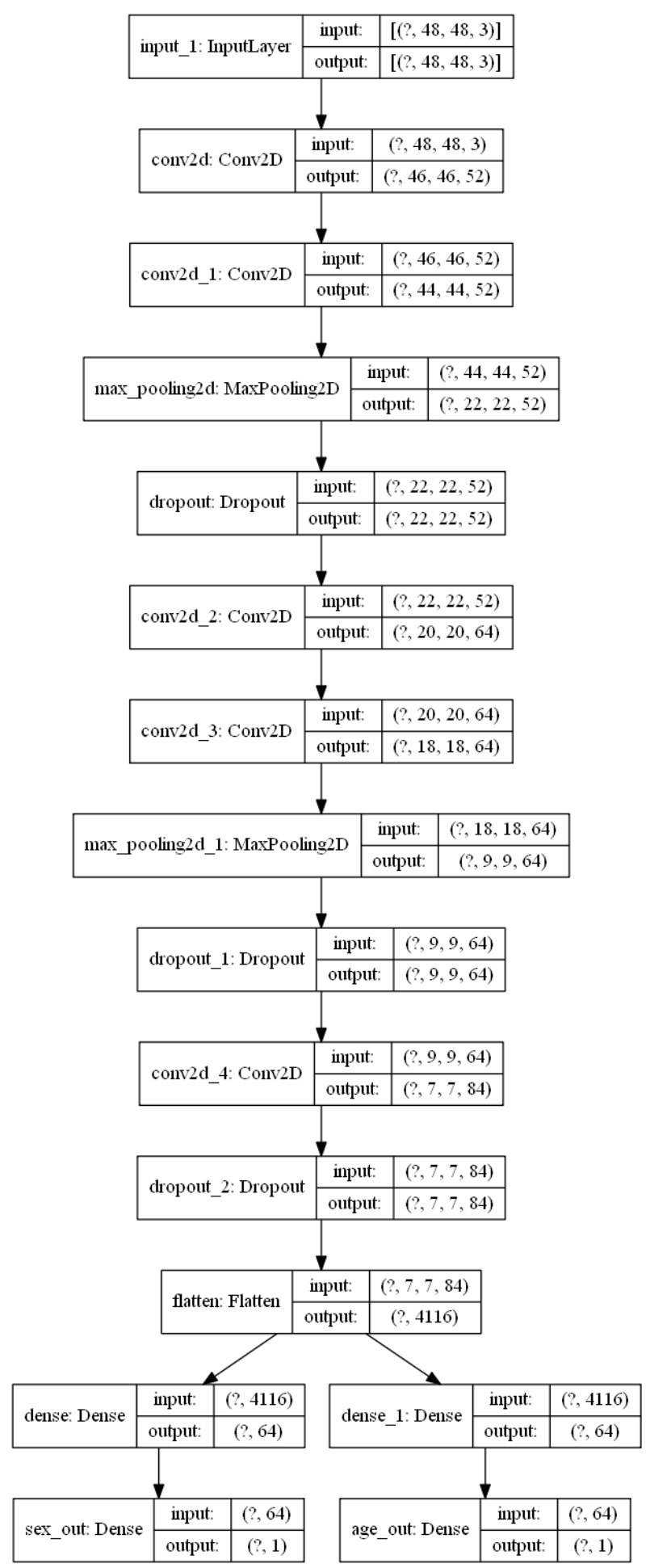

Fig. 4 Algorithm Design

\section{IMPLEMENTATION}

\section{A. Data Gathering}

This is the first step taken in the project. Data plays a very essential role in any deep learning model; hence the source and quality of data proves to be of great importance. In our case, our data is a set of facial images with their age and gender values. We have made use of two datasets.

Published By:

Blue Eyes Intelligence Engineering

and Sciences Publication

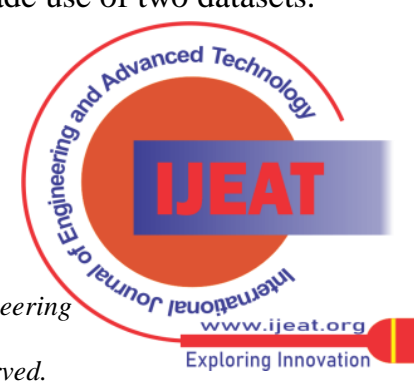


The first one is UTKface dataset. This dataset contains over 20,000 images. They contain data with a wide range of ages (1-116). Some of the other categories present in the dataset are, facial expression, pose, illumination etc. In our project, we will be using only age and gender values. This dataset has a fairly even distribution of gender. $47.7 \%$ of the images are male and the rest are of female images. The age is also wisely distributed throughout the dataset. We have a figure showing the visualization of the age ranges present in the dataset. We have the 20-30 age group take up comparatively higher percentage data of about $32.8 \%$ and very old age group $(80+)$ take up minimal percentage of data of about $2.28 \%$, but considering the actual age percentages in the population today, this representation seems fair. Next, we are using the Fairface dataset. FairFace is a dataset of face images which is race balanced. It contains 108,501 images from 7 different race groups: White, Black, Indian, East Asian, Southeast Asian, Middle Eastern, and Latino. Images were collected from the YFCC-100M Flickr dataset and labeled with race, gender, and age groups. Our project is mainly built to be used by Indian shelter homes and hence, we have tried to enhance the prediction accuracy for Indian population by extracting images with Indian race and combing this with the UTKface dataset to form our final dataset. As we can see in the figure mentioned below, the percentage of Indian images is highest in UTKFace and Fairface dataset and hence, those datasets were considered for the paper.

\section{B. Dataset Preparation}

Though we are considering a dataset which gives us preprocessed images, they can't be used directly to our model. We need to make certain changes to the data to make it compatible to the model. First, we are converting the images to pixel array to be used by the model. We are also resizing the images to $(48,48,3)$ to fit our model and the default size of the UTKFace images are $(200,200,3)$. We are the splitting the data to train and test by a proportion of 0.33 . The y values also have to extracted from the dataset and converted to vector format to be fed into the model.

The input test image that we input from the user also has to pass through a few preprocessing steps to be made compatible for predicting. First, we are adding a padding of white around the image to allow it for the image face detecting and cropping extremities. Next, we are passing the image through the image face detection algorithm where the image face is detected. We then go on to crop the face from the entire image. This will then be resized to $(48,48,3)$ and then converted to the pixel array to be fed into the model for age and gender prediction.

\section{Model Preparation}

In this project, we are using the CNN model to build a custom architecture of 7 layers, of which, we have 5 convolutional layers and 2 fully connected layers. Initially we have an Input layer accepting input image of size $(48,48,3)$. We will then be passing on to the first convolutional layer with 52 nodes and kernel size 3 . The activation function we have used for these layers are ReLu. We have repeated this layer, followed by a maxpool layer with kernel size 2. Pooling mainly helps in extracting sharp and smooth features. It is also done to reduce variance and computations. We then have a dropout layer with proportion 0.3 which is again used to reduce variance and computations. After repeating these 4 layers (with 64 nodes followed by a conv layer of 84 nodes), we then flatten them and connect them to two fully connected layers with 64 units each. Following this, the model has two final output layers, one for age, with ReLu activation (for regression) and the other for gender with sigmoid activation (for classification) function. This model is fit on the dataset, and are trained for 60 epochs. The loss reduction was not significant after 50 epochs and hence was stopped at 60 . The

\section{Web Interface}

The web application is Implemented using Django and MongoDB. Django is a Python-based free and open-source web framework that follows the model-template-views architectural pattern. The models are nothing but MongoDB collections that will accept all the input data. Templates are the html pages which are a collection of forms, login pages, dashboards, etc. Views are the python custom functions that are written for describing functionality of each view of the Django application. MongoDB is classified as a NoSQL database program, MongoDB uses JSON-like documents with optional schemas. This makes this database extremely flexible to use for projects which involve large amounts of data, making it easy to save and navigate through this data.

Once the form input from the user is received, the details are saved in a collection in the database.

The admin, once logged in, can see all the form entries by the user and can accept or reject these. Once accepted, the model is run for that image and the person is allocated to the appropriate shelter home. The admin can also admit the person and run the model after which the person will be allocated in a shelter home. The age, gender and the name of the person will be saved into the respective shelter home collection in the database. These details can later be accessed by the admin for future purposes.

\section{RESULT AND DISCUSSION}

The paper proposed an Age and Gender prediction method using CNN which is used to allot shelter home for homeless people.

Result analysis on the combinational dataset (i.e., Fairface and UTKface dataset) shows an achievement of accuracy 0.8729 for Gender and the loss values of 0.2782 for Gender is as mentioned in table 1

The graph in figure represents a loss history graph. It represents number of epochs on $\mathrm{x}$ axis and loss on the $\mathrm{y}$ axis.

The blue curve represents the train data and the orange curve represents the test data. The train data shows an increase in loss at the starting but decreases gradually as the number of epochs increases. The test data shows ups and downs throughout the process, but also to add it increases first and later decreases. The table I shows the RMSE (Root Mean Square Error) and MAE (Mean Absolute Error) values obtained with respect to age prediction.

The model takes around 54 seconds to evaluate which is approximately 5 millisecond per sample.

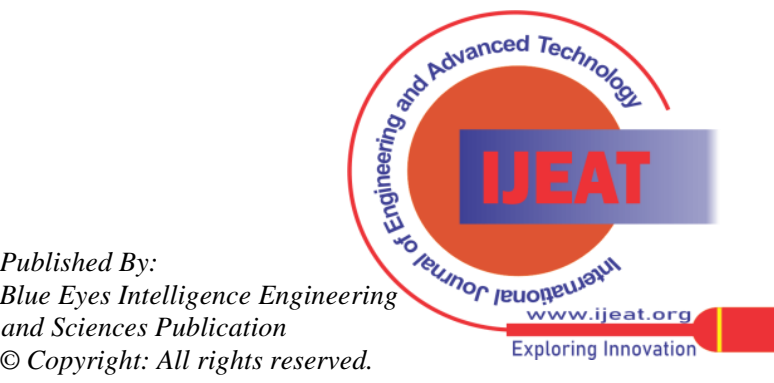


Table- I: RMSE and MAE values with respect to Age Prediction

\begin{tabular}{|c|c|}
\hline Parameters & Value \\
\hline RMSE & 9.5 \\
\hline MAE & 6.7 \\
\hline
\end{tabular}

Table- II: Result Analysis of Gender Estimation

\begin{tabular}{|c|c|}
\hline Parameters & Value \\
\hline Accuracy & 0.8729 \\
\hline Loss & 0.2782 \\
\hline
\end{tabular}

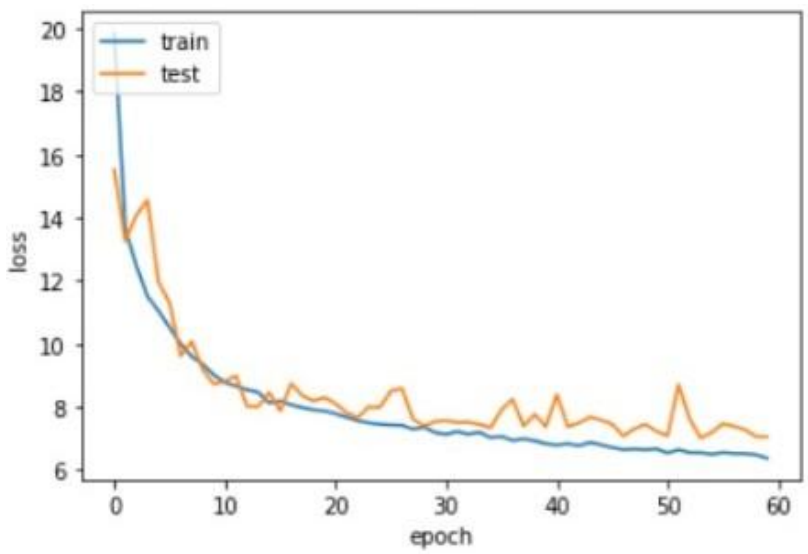

Fig. 5 Graphical Representation of Loss History

\section{CONCLUSION \& FUTURE ENHANCEMENT}

The increase in the number of homeless people in the recent years has led to the difficulty in quick and accurate allocation of these people to the respective shelter homes. The lack of required identification documents is an added difficulty. To overcome this problem, and to help reduce the manual work gone in allocation and paper work preparation, this paper proposes a system that automatically allocates people to various shelter homes based on the facial images. Age and Gender of the person is determined using their facial image. The algorithm used for this purpose is Convolutional Neural Network and Haar Cascade Classifier Algorithm is used for face detection. The model was trained on the dataset which was prepared by combining the UTKFace dataset and extracting only Indian race images from the Fairface dataset. Based on the age and gender values received, they are further allocated to the right shelter home.

A real time fully automated photo searching and needy people detection system could be developed. Many homeless people might have various diseases or sensitive health conditions requiring special care, as a part of future enhancement, the current system can be made compatible to allocate shelter homes based on these conditions also.

\section{REFERENCES}

1. Ari Ekmekji, "Convolutional Neural Networks for Age and Gender Classification" in Stanford University California, USA,2015.

2. G.Levi and T. Hassncer, "Age and gender classification using convolutional neural networks," in Proceedings of the 2015 IEEE Conference on Computer Vision and Pattern Recognition Workshops (CVPRW), pp. 34-42, Boston, MA, USA, June 2015.

3. Ke Zhang, Member, IEEE, Ce Gao, Liru Guo, Miao Sun, Student Member, IEEE, Xingfang Yuan, Student Member, IEEE, Tony X. Han, Member, IEEE, Zhenbing Zhao, Member, IEEE and Baogang Li” Age Group and Gender Estimation in the Wild with Deep RoR Architecture" IEEE August 2017.
4. Eirikur Agustsson, Radu Timofte, Sergio Escalera, Xavier Baro, Isabelle Guyon, Rasmus Rothe" Apparent and real age estimation in still images with deep residual regressors on APPA-REAL database" Open University of Catalonia, Barcelona, Spain, 6 ChaLearn, California, USA,2017.

5. Khurram Zeeshan Haider, Kaleem Razzaq Malik, Shehzad Khalid, Tabassam Nawaz, Sohail Jabbar "Deepgender: real-time gender classification using deep learning" 10 March 2017, Accepted on 24 August 2017

6. X. Liu, J. Li, C. Hu and J. Pan, "Deep convolutional neural networks-based age and gender classification with facial images," 2017 First International Conference on Electronics Instrumentation \& Information Systems (EIIS), Harbin, 2017.

7. Aakash Rastogi, Manish Aneja, Narina Thakur, Mayur Dhingra, Naman Gupta. "Gender Classification from Facial Images-Performing the classification of Gender from the facial Images and implementing the same on the Real Time" International Journal for Research in Applied Science \& Engineering Technology (IJRASET) Volume 6 Issue VI, June 2018

8. Smith, Philip \& Chen, Cuixian. (2018). Transfer Learning with Deep CNNs for Gender Recognition and Age Estimation. 2564-2571. 10.1109/BigData.2018.8621891.

9. Kunal Jain, Muskan Chawla, Anupma Gadhwal, Rachna Jain and Preeti Nagrath. "Age and Gender Prediction Using Convolutional Neural Network", Proceedings of First International Conference on Computing, Communications, and Cyber-Security (IC4S 2019)

10. Insha Rafique, Awais Hamid, Sheraz Naseer, Muhammad Asad, Muhammad Awais and Talha Yasir. "Age and Gender Prediction using Deep Convolutional Neural Networks", International Conference on Innovative Computing (ICIC), 2019

11. Ishita Verma, Urvi Marhatta, Sachin Sharma, Vijay Kumar. "Age Prediction using Image Dataset using Machine Learning” International Journal of Innovative Technology and Exploring Engineering (IJITEE) ISSN: 2278-3075, Volume-8 Issue-12S3, October 2019

12. B. Lee, S. Z. Gilani, G. M. Hassan and A. Mian, "Facial Gender Classification - Analysis using Convolutional Neural Networks," 2019 Digital Image Computing: Techniques and Applications (DICTA), Perth, Australia, 2019

13. Trivedi, Gangesh \& Pise, Nitin. (2020). Gender Classification and Age Estimation using Neural Networks: A Survey. International Journal of Computer Applications. 176. 975-8887.

14. 14. Huynh, H.T., Nguyen, H. Joint Age Estimation and Gender Classification of Asian Faces Using Wide ResNet. SN COMPUT. SCI. 1, 284 (2020)

15. Bulbul Agrawal (\&) and Manish Dixit. "Age Estimation and Gender Prediction Using Convolutional Neural Network", Proceedings in Adaptation, Learning and Optimization book series (PALO, volume 13),2020.

\section{AUTHORS PROFILE}

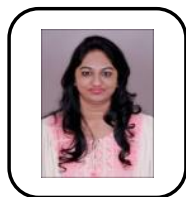

Shravya Suresh, Student, Computer Science and Engineering, Bangalore Institute of Technology, Bengaluru, India. Email: shravya995@gmail.com

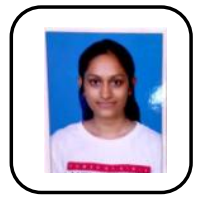

Sneha Venkatesh, Student, Computer Science and Engineering, Bangalore Institute of Technology, Bengaluru, India. Email: snehavenkatesh10@gmail.com

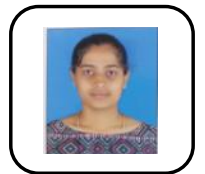

Vidya Shree S, Student, Computer Science and Engineering, Bangalore Institute of Technology, Bengaluru, India. Email: vidyashree7499@gmail.com

Published By: Blue Eyes Intelligence Engineering and Sciences Publication (C) Copyright: All rights reserved.

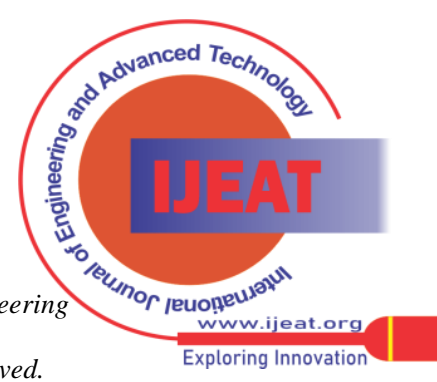


Age and Gender Based Organisation of Shelter Homes using Convolutional Neural Networks

Hemalatha V R, Student, Computer Science and Engineering, Bangalore Institute of Technology, Bengaluru, India. Email: hemaprasad1899@gmail.com

Dr. T Vijaya Kumar, Associate Professor, Computer Science and Engineering, Bangalore Institute of Technology, Bengaluru, India. Email: tvijaykumar@bit-bangalore.edu.in

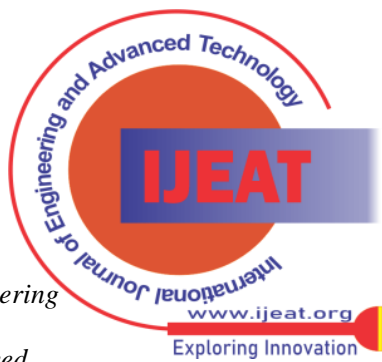

